\title{
Editorial
}

\section{Dying for a living: plants do it too}

\author{
Bernard Rubinstein ${ }^{1,3}$ and Barbara A. Osborne ${ }^{2}$ \\ 1 Department of Biology, University of Massachusetts, Amherst, MA 01003, \\ USA \\ 2 Department of Veterinary and Animal Science, University of Massachusetts, \\ Amherst, MA 01003, USA \\ 3 Corresponding author: B. Rubinstein
}

The irony that cell death is necessary for the survival of an organism is as valid for plants as it is for animals. However, plant systems that require cell death generally have little in common with those in animals. This leads one to question whether cell death pathways in plants and animals have any similarities among them. The reviews that follow describe some developmental systems that are peculiar to plants and for which cell death is an integral component. Also discussed are mechanisms leading to cell death and how these processes might relate to what is known about animals.

One way to obtain an overview of the role of programmed cell death in plants is to consider that only particular cells of a tissue may die, or that there may be an extensive annihilation of cells, leading to degradation of an entire organ. The death of single cells or small groups of cells in plants may be a part of normal development as is the case in animals. In his review, Beers (1997) points out the exquisite controls that must operate when only particular cells of the plant associated with reproduction or vegetative growth are signalled to die, whereas adjoining cells continue to differentiate and function normally. An illustration of this phenomenon is described by Fukuda (1997); certain cells of the vascular system must die before they can operate as transporters of water and minerals. And here is another irony peculiar to plants. The program for death during vascular cell differentiation has not evolved to eliminate cells that have fulfilled their purpose, but to 'design' cells for their eventual function.

In some cases, the death of small groups of cells is not a normal part of development, but only occurs as a response to certain stresses, such as infection. And here, yet another irony presents itself. The genetic program that causes cells to die after sensing the presence of a pathogen, actually appears to help stymie spread of the disease. Aspects of this very interesting and intensively investigated plant response are discussed by Morel and Dangl (1997) and by Gilchrist (1997).

In contrast to situations where death occurs only in selected cells of a tissue, there are many examples in plants in which whole organs, such as leaves, flowers and fruits may die. Unlike animals, most plants continue to grow and reproduce during their entire lifetimes, so geneticallybased programs have evolved that sense when an organ is no longer needed, with the result that there is a massive, synchronous cell suicide. As discussed by Hadfield and Bennett (1997), these pathways to death may take days or weeks, but in the case of certain flowers, death of the entire organ may begin only hours after opening. Closely superimposed on the cell death response, is the retrieval of materials from the senescing cells. The plant effectively parasitizes its own organs during their death, so that the nutrients can be used for the production of new tissues.

The mechanisms leading to plant cell death and their comparison to animal systems are dealt with by all of the reviewers. While caspases, so crucial to animal cell death, have not been definitively identified in plants, other proteases and the ubiquitin system may play important roles, as pointed out by Beers (1997) and Fukuda (1997). Morel and Dangl (1997) refer to reactive oxygen species and discuss the possibility that they originate from mechanisms similar to the oxidative burst in animals. Changes in the nucleus and nuclear material that appear to resemble those in animal cells are illustrated by Gilchrist (1997), and he also points out the intriguing similarity of certain mycotoxins to sphinganine and proposes a role for these toxins in ceramide metabolism of the plant. Hadfield and Bennett (1997) discuss the hormonal signals as well as the genetic and biochemical determinants that cause senescence and death of entire organs.

As we attempt to compare pathways of cell death between plant and animal systems, some important differences between the two must be kept in mind. Along with the very different life cycles, developmental processes and anatomy, there are critical distinctions at the cell level. Plant cells are surrounded by a chemically complex and often rigid cell wall. The wall may be a source of signals leading to resistance to predation and disease, and wall 'softening' accompanies many senescence processes, with fruits being an extreme example. Secondly, 80 to $90 \%$ of the volume of a mature plant cell is comprised of the vacuole, an organelle containing a potentially deadly mixture of hydrolytic enzymes at a low $\mathrm{pH}$. A loss of differential permeability of the tonoplast, the membrane surrounding the vacuole, would lead to severe disruption of metabolic events, if not cell death.

Keeping these differences in mind, the reader should find very interesting the systems that are unique to plants and that depend on tighly regulated cell death programs. Determining the components of these programs will not only aid in understanding plant development, but may lead to improvements in the abilities of plants to resist predation and disease, as well as to gains in crop production and food preservation. An invaluable manner of acquiring the necessary information is to determine if cell death pathways or particular components of pathways are conserved between plants and animals. With this series of reviews, we intend to stimulate debate and conversation between plant and animal cell death research teams. 
Dying for a living: plants do it too B Rubinstein and BA Osborne

\section{References}

Beers EP (1997) Programmed cell death during plant growth and development. Cell Death Differ. 4: 649-661

Fukuda $\mathrm{H}$ (1997) Programmed cell death during vascular system formation. Cell Death Differ. 4: 684-688
Gilchrist DG (1997) Mycotoxins reveal connections between plants and animals in apoptosis and ceramide signaling. Cell Death Differ. 4: 689-698

Hadfield KA and Bennett AB (1997) Programmed senescence of plant organs. Cell Death Differ. 4: $662-670$

Morel J-B and Dangl JL (1997) The hypersensitive response and the induction of cell death in plants. Cell Death Differ. 4: 671-683 